\title{
A doubly hermaphroditic chiral crown ether $\dagger$
}

Cite this: CrystEngComm, 2014
5984
Received 24th April 2014,
Accepted 23rd May 2014
DOI: 10.1039/c4ce00879k
www.rsc.org/crystengcomm

A single-crystal structure determination on the S-protected form of a chiral 18-crown- 6 derivative known to be a selective catalyst for thiolysis reactions of amino acid derivatives has shown the molecule to crystallise in an unsolvated form where the macrocyclic ring has a conformation in which the dipoles of substituent amide units are aligned parallel. The resulting polar entities are linked through $\mathrm{NH} \cdots \mathrm{O} \mathrm{H}$-bonds and weaker interactions which can be considered to result in doubly hermaphroditic links, the whole crystal proving to be polar. The possible consequences of the observed secondary interactions, some being intramolecular, are considered in relation to the mechanism of catalysis by the isolated molecule.

\section{Introduction}

Catalysis is one of the most fundamental reflections of the importance of supramolecular chemistry, since the interactions of a catalyst with substrates, intermediates, transition states and products must all be labile and have as their origin the numerous forms that intermolecular forces may take. ${ }^{1}$ Asymmetric catalysis is a particularly important aspect of this field, ${ }^{2}$ in part because of its significance in the understanding of enzyme action, ${ }^{3}$ and one of the families of molecules (in forms modified by the introduction of chirality) long considered useful in developing a detailed analysis of asymmetric transformations is that of the crown ethers. ${ }^{4,5}$ Nonetheless, in regard to substrate binding by chiral macrocycles in general, there remains a need for a more profound understanding of the operative factors. ${ }^{6}$ In the belief that a single-crystal structure determination of the chiral crown ether A (Fig. 1), the S-protected form of a highly discriminatory

\footnotetext{
${ }^{a}$ Stratingh Institute for Chemistry, University of Groningen, Nijenborgh 7 , 9747 AG, The Netherlands.E-mail: a.k.h.hirsch@rug.nl

${ }^{b}$ UMR 7177 du CNRS, Institut de Chimie, Université de Strasbourg,

Strasbourg, France

${ }^{c}$ Institut de Science et d'Ingénierie Supramoléculaires, Université de Strasbourg, Strasbourg 67083, France. E-mail: harrowfield@unistra.fr

$\dagger$ CCDC 997723. For crystallographic data in CIF or other electronic format see DOI:10.1039/c4ce00879k
}

thiolysis catalyst, ${ }^{7}$ might reveal its substrate binding interactions, the present work was conducted. The 18-crown-6 framework which forms the core of $\mathbf{A}$ has been of particular interest for the catalysis of reactions involving protonated primary amines (such as amino acids) because of the efficacy with which its sixfold-symmetric conformation may serve as a binding site for $\mathrm{RNH}_{3}{ }^{+}$, although it is well-known that this conformation is not necessarily that of the unbound ligand. ${ }^{4,5}$ Interestingly, A crystallises readily in an unsolvated form, i.e. without any form of bound species present and in a conformation which is not optimal for binding $\mathrm{RNH}_{3}{ }^{+}$ species, its structure illustrating the forces that may be in balance in complex formation and indicating a possible mechanism for guest expulsion from any complex.

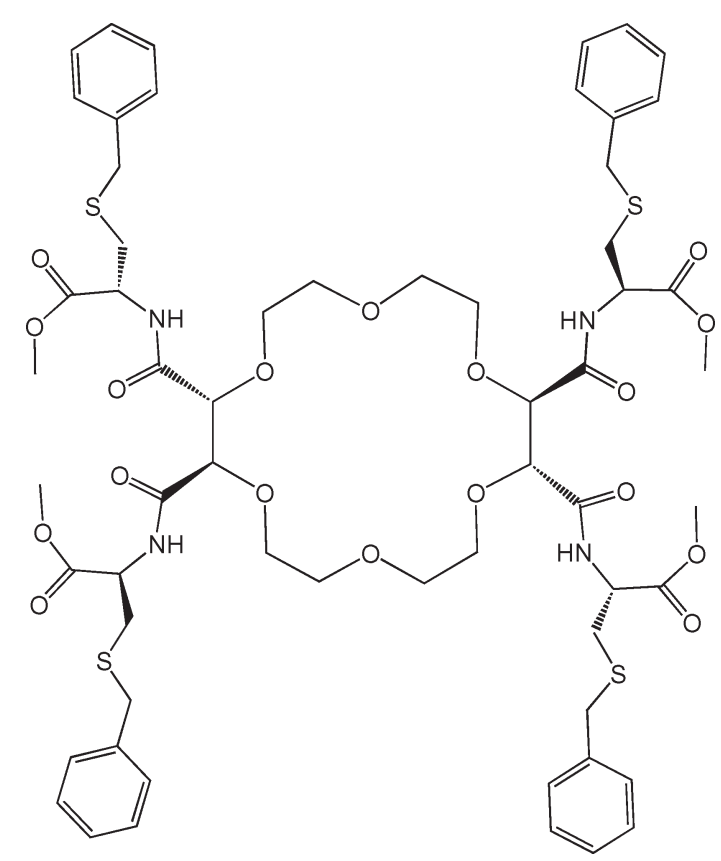

Fig. 1 The crown ether $\mathrm{A}$, derived from $R, R$-tartaric acid and with side chains derived from $R$-cysteine. 


\section{Experimental}

Synthesis

A was a sample available from earlier work ${ }^{7}$ and was crystallised for the X-ray diffraction measurements by slow evaporation of its solution in dichloromethane.

\section{Crystallography}

The structure determination was performed by Dr Lydia Brelot at the Service de Radiocristallographie of the University of Strasbourg. The crystals were placed in oil and a single one selected, mounted on a glass fibre and placed in a lowtemperature $(173(2) \mathrm{K})$ nitrogen stream. The X-ray diffraction data were collected on a Nonius-Kappa CCD diffractometer using graphite-monochromated MoK $\alpha$ radiation $(\lambda=0.71073 \AA)$ and a "phi-scan" scan mode. COLLECT ${ }^{8}$ software was used for the data measurement and DENZO-SMN ${ }^{9}$ for the processing. The structure was solved by direct methods using SHELXS-97. ${ }^{10}$ The refinement and further calculations were carried out using SHELXL-97. ${ }^{11}$ CCDC 997723.

Crystal data: triclinic, space group $P 1, a$ 9.3854(5), $b$ 14.0773(6), c 14.0972(6) ̊, $\alpha$ 65.540(2), $\beta$ 70.737(2), $\gamma 79.985(2)^{\circ}, 173(2) \mathrm{K} ; V$ 1598.95(13) $\AA^{3}, F(000) 672, D_{\mathrm{c}}(Z=1)$ $1.318 \mathrm{~g} \mathrm{~cm}^{-3}, \mu_{\mathrm{Mo}} 0.221 \mathrm{~cm}^{-1}$, specimen $0.50 \times 0.14 \times 0.04 \mathrm{~mm}$, $2 \theta_{\max } 55^{\circ}, N_{\mathrm{t}} 16352, N 11142\left(R_{\text {int }} 0.101\right), N_{\mathrm{o}} 8232, R_{1} 0.072$, $\mathrm{w} R_{2} 0.168, S 1.080$.

\section{Results and discussion}

As noted above, the crown ether A crystallises from dichloromethane in an unsolvated form. As is usually the case in crystal structures of uncomplexed 18-crown-6 and its derivatives, ${ }^{4,7}$ the macrocyclic ring conformation is not of (effective) sixfold symmetry with oxygen lone pairs directed towards the centre of the ring. In fact, the conformation found is similar to that of as simple a species as 1,4-dithia7,10,13,16-tetraoxacyclo-octadecane, ${ }^{12}$ with two adjacent oxygen atoms everted, as are the two sulfur atoms in the simple crown. Unsurprisingly, it is also very similar to that of the crown ring in the closely related species ${ }^{13}$ in which phenylalanine units replace those of cysteine in $\mathbf{A}$, although there are significant differences in the crystal lattices of these two compounds (see ahead). The two amide arms of a given tartrate unit of A project in opposite directions essentially perpendicular to the mean plane of the macrocyclic ring, resulting for the whole molecule in pairs of adjacent arms lying to each side and ensuring that the chirality is expressed in three dimensions (Fig. 2).

From the Hirshfeld surface ${ }^{14}$ for molecule $\mathbf{A}$ in its crystal (Fig. 3), as obtained by the application of CrystalExplorer, ${ }^{15}$ interactions between separate molecules which exceed simple dispersion forces appear to be dominated by H-bonding, although other interactions are certainly apparent.

Intermolecular amide-NH $\cdots \mathrm{O}$-carbonyl interactions

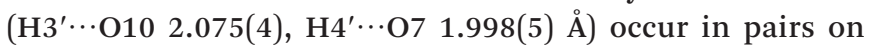
opposite sides of the molecules, leading to the formation of a

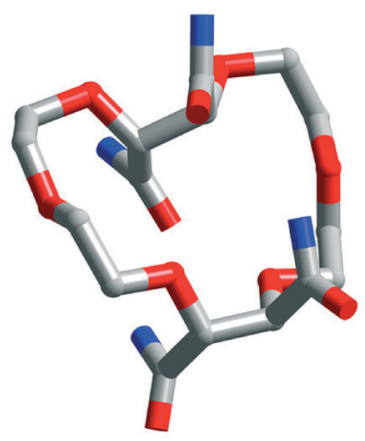

(a)

(b)

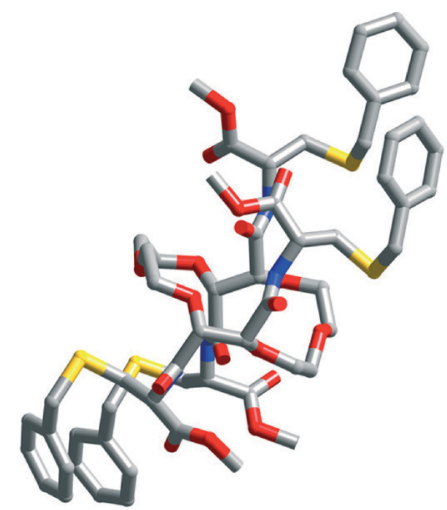

(b)
Fig. 2 Views of the molecule $A$ as found in its crystalline lattice: (a) the macrocyclic (crown ether) ring with its substituents truncated to the amide units; (b) the full molecule, showing how the substituents form pairs oriented to opposite sides of the macrocyclic ring. $\mathrm{C}=$ gray, $\mathrm{N}=$ blue, $\mathrm{O}=$ red, $\mathrm{S}=$ yellow; for clarity, $\mathrm{H}$-atoms are not shown.

polymeric chain running parallel to the $a$-axis (Fig. 4(a)). What the Hirshfeld surface does not reveal is that there are similar intramolecular H-bonds ( $\mathrm{H} 1 \cdots \mathrm{O} 16$ 2.187(5), H2 $\cdots \mathrm{O} 13$ $2.378(5) \AA$ ) also essentially oriented along the $a$-axis (Fig. 4(b)). The importance of these intramolecular H-bonds is that they are the result of the carbonyl units of all the tartramide substituents being oriented in the same sense (associated with the particular conformation of the macrocyclic ring), thus causing each molecule to be polar and the dipoles in the chain to be oriented in a head-to-tail fashion, making the whole chain polar. This (see ahead) is of significance in relation to the fact that the crystal is also polar, ${ }^{16}$ the space group being $P 1$.

A further dissection of the lattice is possible on recognising that the molecules form another type of head-totail polymeric array (Fig. 5) in columns running parallel to the $b$-axis. This array involves a "double-hermaphrodite" linkage and thus a polymeric form of hermaphroditic interaction, ${ }^{17}$ the partial mutual penetration of adjacent molecules appearing to be the result of $\mathrm{CH}^{\cdots} \pi \pi$ interactions involving the methyl groups of the amino acid ester units $\left(\mathrm{C} 57 \cdots \mathrm{H} 40 \mathrm{C}^{\prime}\right.$

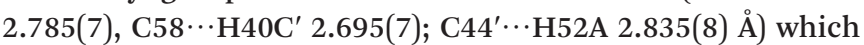
are seen as less intense red regions of the Hirshfeld surface. Again, this chain is polar, since the molecules all have the same orientation, although presumably the dipole in the $a$ direction due to the H-bonding array would be greater.

To create a polar crystal from polar 1D chains, it is necessary to link the chains so that they have a common direction. ${ }^{16}$ In the present case, taking the $\mathrm{H}$-bonded chain as a primary component, links in the $b$ direction are those which result in the hermaphroditic units and it is the double characteristic of these which results in a common orientation. In the $c$ direction, while there is no obvious interpenetration, there are reciprocal interactions of the $\mathrm{CH} \cdots \pi$ type (Fig. 6) involving the crown-ring methylene groups which apparently 

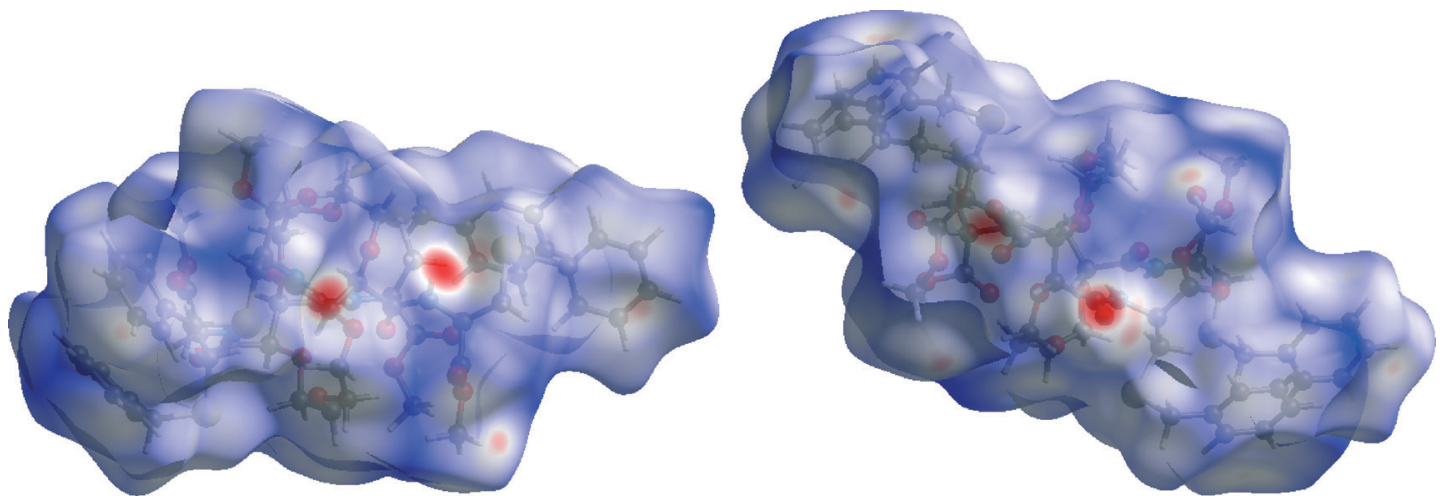

Fig. 3 Views of the Hirshfeld surface for molecule A in its crystal lattice, showing the two sides from which $\mathrm{H}$-bonds extend. By convention, red areas of the surface denote regions where nuclei within the surface come into closer contact with nuclei in other molecules outside the surface than can be attributed to dispersion forces alone.

(a)

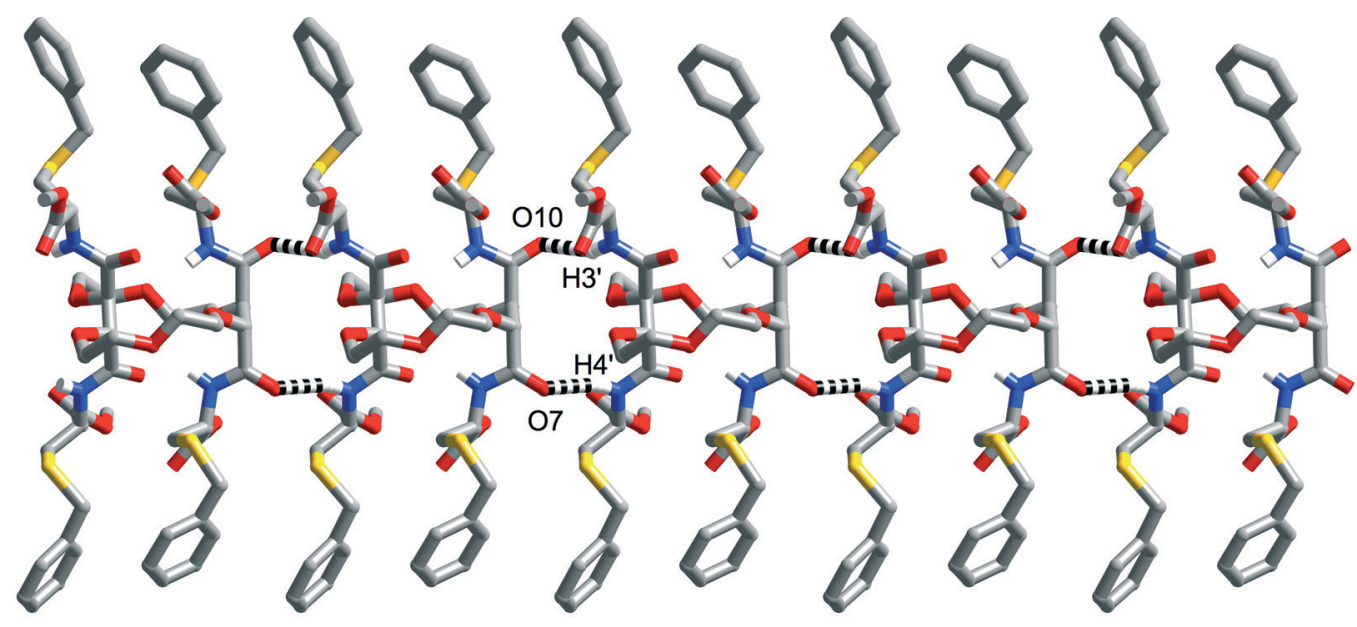

(b)

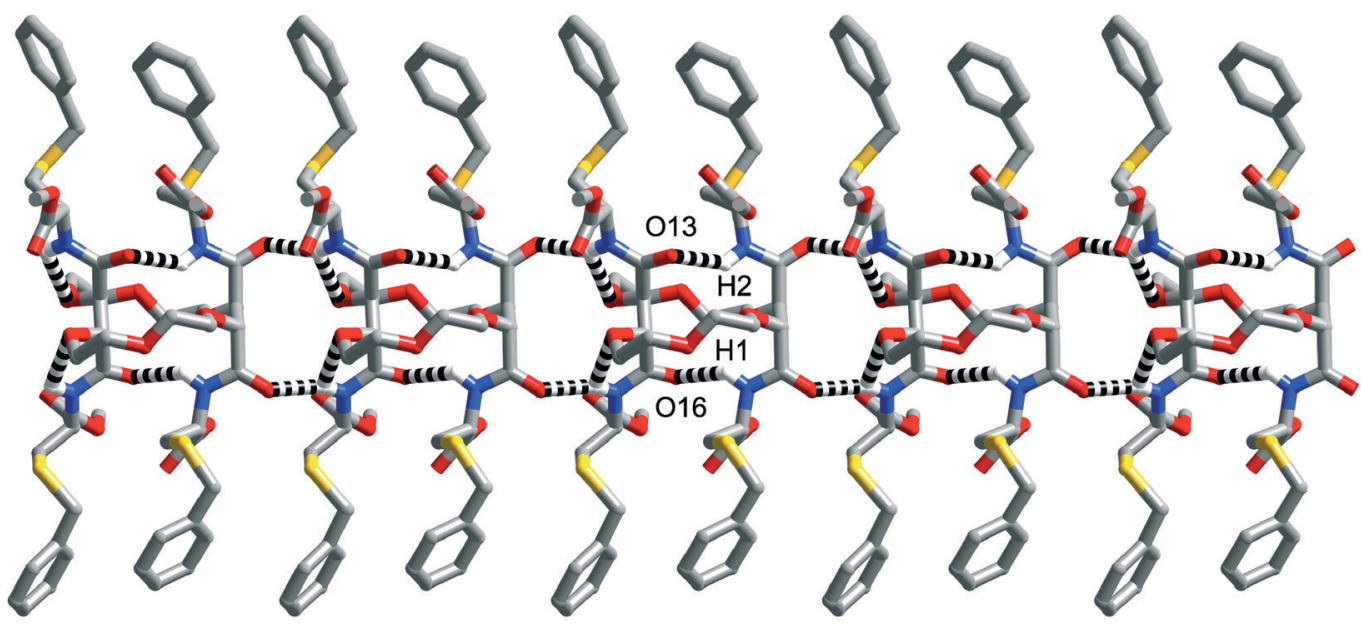

Fig. 4 Views of the $\mathrm{H}$-bonded chains of molecules of A running parallel to the a-axis, showing (dashed lines) (a) the intermolecular interactions and (b) the inter- and intra-molecular interactions. $\mathrm{H}$-atoms are shown on $\mathrm{N}$ only.

favour the repetition of a common orientation. These interactions could of course not be present in the active catalyst species obtained by removal of the benzyl groups from the S-centres. The subtlety of factors determining whether or not 


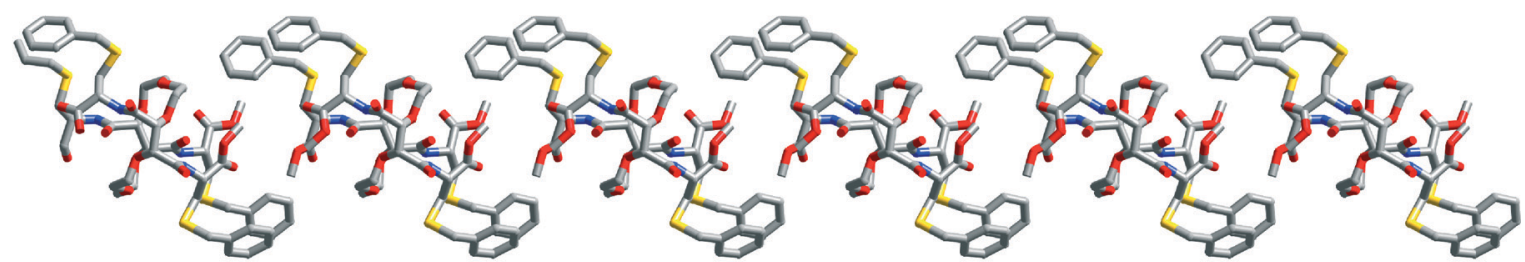

(a)

\section{(b)}

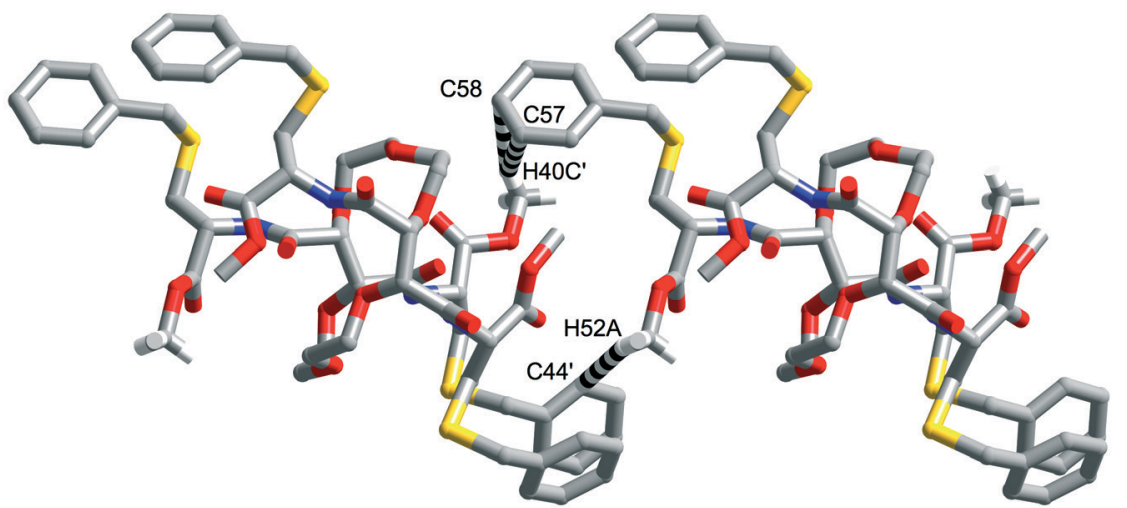

Fig. 5 (a) Partial view of the chain of "double-hermaphrodite" interactions running parallel to the $b$-axis; (b) view of one adjacent pair showing the $\mathrm{CH} \cdots \pi$ contacts (dashed lines) apparently favouring the interpenetration.

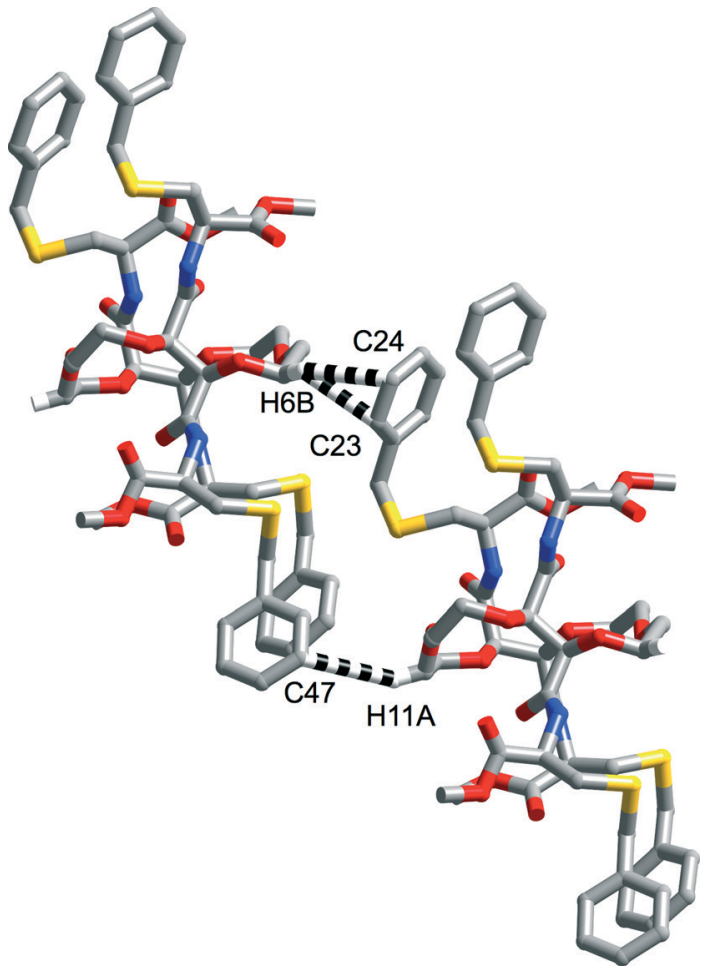

Fig. $6 \mathrm{CH}$ - aromatic contacts apparent between adjacent molecules

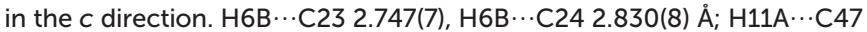
3.079(8) A. the crystal is polar is illustrated by the fact that the crystal formed by the phenyalanine analogue of $\mathbf{A}$ is not polar, ${ }^{13}$ despite the presence of similar $\mathbf{H}$-bonded chains as in $\mathbf{A}$.

\section{Conclusions}

The structure described herein is clearly one of a molecule which cannot be considered "preorganised" for its catalytic functions in the sense that the crown ether ring conformation is not optimised for binding to the three protons of an $\mathrm{RNH}_{3}{ }^{+}$species. Various structure determinations ${ }^{18}$ of aminoacid-substrate adducts of ligands closely related to A do, however, reveal the presence of this conformation, showing that the energy required to attain it is unlikely to be prohibitive in the case of A. Assuming that the intramolecular $\mathrm{NH}^{\cdots} \mathrm{O}$ interactions seen here (and elsewhere ${ }^{13}$ ) are the primary factor influencing the unsymmetrical conformation, they can be seen as a means of regulating the affinity of the catalytic site for both substrates and products. Expulsion of a product retaining the ammonium unit required for binding to the catalyst could be crucial to the catalyst efficiency, thus it is important that some entasis may be involved in the initial substrate binding via the same mechanism as would be involved in product retention. A secondary factor influencing the accessibility of the active site could be the aggregation seen in the lattice of crystalline A which leads to the doubly hermaphroditic chains. These add to the factors which have been deduced to 
be important influences on catalyst function on the basis of substrate complex structures. ${ }^{1,18 d}$

\section{Acknowledgements}

A. K. H. Hirsch gratefully acknowledges support by a postdoctoral fellowship of the Human Frontier Science Program (HFSP).

\section{References}

1 (a) J.-M. Lehn, Supramolecular Chemistry - Concepts and Perspectives, VCH, Weinheim, 1995, ch. 5, in particular; (b) see also J.-M. Lehn, Chem. Soc. Rev., 2007, 36, 151-160.

2 (a) E. N. Jacobsen, A. Pfaltz and Y. Yamamoto, Comprehensive Asymmetric Catalysis: Supplement 1, Springer-Verlag, Berlin, 2004; (b) M. Crego-Calama, Supramolecular Chirality, Top. Curr. Chem., ed. D. N. Reinhoudt, Springer-Verlag, Berlin, 2006, vol. 265.

3 A. Warshel, Annu. Rev. Biophys. Biomol. Struct., 2003, 32, 425-443.

4 (a) R. M. Izatt, C. Y. Zhu and J. S. Bradshaw, in Crown Compounds - Towards Future Applications, ed. S. R. Cooper, VCH, Weinheim, 1992, ch. 12, pp. 207-233; (b) J. S. Bradshaw, R. M. Izatt, A. I. Bordunov, C. Y. Zhu and J. K. Hathaway, in Comprehensive Supramolecular Chemistry, ed. J. L. Atwood, J. E. D. Davies, D. D. Macnicol and F. Vögtle, Elsevier Science, Oxford, 1996, vol. 1 (volume editor G. W. Gokel), ch. 2, pp. 35-95.

5 G. W. Gokel, Crown Ethers and Cryptands, Monographs in Supramolecular Chemistry No. 3, ed. J. F. Stoddart, Royal Society of Chemistry, Cambridge, 1991, ch. 4 in particular.

6 (a) A. Gualandi, L. Cerisoli, H. Stoeckli-Evans and D. Savoia, J. Org. Chem., 2011, 76, 3399-3408; (b) S. E. Denmark, N. D. Gould and L. M. Wolf, J. Org. Chem., 2011, 76, 4337-4357.

7 (a) J.-M. Lehn and C. Sirlin, J. Chem. Soc., Chem. Commun., 1978, 949-951; (b) J.-M. Lehn and C. Sirlin, New J. Chem., 1987, 11, 693-702.
8 R. W. W. Hooft, COLLECT Data Collection Software, Bruker AXS, Delft, The Netherlands, 2008.

9 Z. Otwinowski and W. Minor, Methods in Enzymology, Macromolecular Crystallography, Part A, Academic Press, New York, 1997, vol. 276, pp. 307-326.

10 G. M. Sheldrick, Acta Crystallogr., Sect. A: Found. Crystallogr., 1990, 46, 467-473.

11 G. M. Sheldrick, SHELXL-97, Universität Göttingen, Göttingen, Germany, 1999.

12 (a) A. J. Blake, C. Radek and M. Schröder, Acta Crystallogr., Sect. C: Cryst. Struct. Commun., 1995, 51, 2668-2771; (b) N. K. Dalley, S. B. Larson, N. S. Smith, K. L. Matheson, R. M. Izatt and J. J. Christensen, J. Heterocycl. Chem., 1981, 18, 463-467.

13 P. Singh, M. L. Miles and S. Davis, Acta Crystallogr., Sect. C: Cryst. Struct. Commun., 1990, 46, 263-267.

14 M. A. Spackman and D. Jayatilaka, CrystEngComm, 2009, 11, 19-32.

15 CrystalExplorer 3.1, S. K. Wolff, D. J. Grimwood, J. J. McKinnon, M. J. Turner, D. Jayatilaka and M. A. Spackman, University of Western Australia, 2012.

16 (a) B. Naranjo, J. K. Gimewski and S. Putterman, Nature, 2005, 434, 1115-1117; (b) R. Glaser, Acc. Chem. Res., 2007, 40, 9-17.

17 L. Brelot, X.-Y. Cao, J. M. Harrowfield, J.-M. Lehn, K. Rissanen and L. Russo, CrystEngComm, 2011, 13, 2346-2349 and references therein.

18 See, for example, (a) B. Chevrier, D. Moras, J.-P. Behr and J.-M. Lehn, Acta Crystallogr., Sect. C: Cryst. Struct. Commun., 1987, 43, 2134-2137; (b) P. J. Dutton, F. R. Fronczek, T. M. Fyles and R. D. Gandour, J. Am. Chem. Soc., 1990, 112, 8984-8985; (c) H. Nagata, H. Nishi, M. Kamigauchi and T. Ishida, Chirality, 2008, 20, 820-827; (d) H. Nagata, Y. Machida, H. Nishi, M. Kamigauchi, K. Minoura and T. Ishida, Bull. Chem. Soc. Jpn., 2009, 82, 219-229. 